\title{
A construction method to study the role of incidence in the adaptive dynamics of pathogens with direct and environmental transmission
}

Kisdi, Eva

2013-03

Kisdi , E \& Boldin , B 2013 , ' A construction method to study the role of incidence in the adaptive dynamics of pathogens with direct and environmental transmission ', Journal of mathematical biology , vol. 66 , no. 4-5 , pp. 1021-1044 . https://doi.org/10.1007/s00285-012-0563-4

http://hdl.handle.net/10138/173657

https://doi.org/10.1007/s00285-012-0563-4

acceptedVersion

Downloaded from Helda, University of Helsinki institutional repository.

This is an electronic reprint of the original article.

This reprint may differ from the original in pagination and typographic detail.

Please cite the original version. 


\title{
A construction method to study the role of incidence in the adaptive dynamics of pathogens with direct and environmental transmission*
}

\author{
Éva Kisdi \& Barbara Boldin
}

This is the Author Version of an article published in the Journal of Mathematical Biology. The final publication is available at Springer Verlag via http://dx.doi.org/10.1007/s00285-012-0563-4.

Please cite as: Kisdi E. \& B. Boldin. 2013. A construction method to study the role of incidence in the adaptive dynamics of pathogens with direct and environmental transmission. J. Math. Biol. 66: $1021-1044$.

\section{Abstract}

We study the adaptive dynamics of virulence of a pathogen transmitted both via direct contacts between hosts and via free pathogens that survive in the environment. The model is very flexible with a number of trade-off functions linking virulence to other pathogen-related parameters and with two incidence functions that describe the contact rates between hosts and between a host and free pathogens. Instead of making a priori particular assumptions about the shapes of these functions, we introduce a construction method to create specific pairs of incidence functions such that the model becomes an optimization model. Unfolding the optimization model leads to coexistence of pathogen strains and evolutionary branching of virulence. The construction method is applicable to a wide range of eco-evolutionary models.

Keywords: adaptive dynamics critical function analysis evolution of virulence evolutionary branching incidence function

Mathematics Subject Classification (2010) 92D15 92D30 92D40

\footnotetext{
*This paper is dedicated to Odo Diekmann on the occasion of his 65th birthday.

${ }^{\dagger}$ Corresponding author: Éva Kisdi, Department of Mathematics and Statistics, University of Helsinki, PO Box 68, 00014 Helsinki, Finland. e-mail: eva.kisdi@helsinki.fi

${ }^{\ddagger}$ Barbara Boldin, Faculty of Mathematics, Natural Sciences and Information Technologies, University of Primorska, Glagoljaška 8, SI-6000 Koper, Slovenia
} 


\section{Introduction}

Many pathogens spread via both direct and environmental transmission, i.e., they can be transmitted either from host to host or via free pathogen particles that survive in the environment. For example, a virus might be picked up from a handshake (direct contact) or from touching a door handle after an infected person (environmental transmission). If the virus dies fast outside the host such that environmental transmission is effective only if it happens within a short time, then the rates of handshake and of touching the same door handle may be combined into one transmission factor (Breban, in press). If, however, free pathogen particles can survive for a non-negligible length of time, then environmental transmission needs to be considered as a distinct transmission mode (Li et al. 2009); this is most obvious when free pathogens outlive the host who produced them. Environmental transmission is important for a number of human pathogens such as influenza and smallpox viruses, Corynebacterium diphtheriae, Mycobacterium tuberculosis, Streptococcus pneumoniae (Walther and Ewald 2004) and cholera (Colwell and Huq 1994; King et al. 2008) as well as for avian influenza (Rohani et al. 2009).

In addition to influencing the dynamics of the epidemics, there is an intriguing reason why direct and environmental transmission need to be distinguished: the pathogen may need contrasting adaptations to improve its transmission when infecting directly and when infecting through the environment. Pathogen strains adapted to alternative transmission modes may coexist while exploiting the same host (Roche et al. 2011), and may evolve from a single ancestral strain via evolutionary branching (Boldin and Kisdi in press). The alternative modes of transmission can thus contribute to the diversity of pathogens.

Whether or not evolutionary branching is possible depends critically on the so-called incidence functions, which describe how the rates of encountering other hosts and encountering free pathogens depend on the population densities of hosts and of pathogens, respectively. The simplest assumption is the mass action law in both transmission modes; this excludes evolutionary branching (Day 2002; Boldin and Kisdi in press; see below). For direct transmission all models cited here assumed mass action, even though standard incidence is often thought to be a better approximation (de Jong et al., 1995). Standard incidence assumes that the rate of encounters between hosts is constant (independent of population size), and the probability that the encountered host is infectious is proportional to the frequency of infected hosts (therefore standard incidence is also called "frequency-dependent transmission"). For environmental transmission, Breban et al. (2010) proposed a hyperbolically saturating incidence function based on an underlying model of how susceptible hosts encounter free pathogens. Roche et al. (2011) used this hyperbolic function, whereas Boldin and Kisdi (in press) showed that evolutionary branching is always possible if the incidence function is concave.

In this paper, we focus on the role of incidence in evolutionary branching. The model we study is very flexible as it contains a number of arbitrary trade-off functions next to 
the two incidence functions. Because of the large number of unspecified functions, finding evolutionary branching points needs to be constructive rather than attempted on the basis of trial and error. A further advantage of the constructive approach is that when it fails, it excludes branching for a certain class of models. Building on the method of critical function analysis (de Mazancourt and Dieckmann 2004; Kisdi 2006; Geritz et al. 2007), we introduce here a constructive method to obtain incidence and trade-off functions that lead to evolutionary branching. First, we show how an environmental incidence function can be constructed to match an arbitrary incidence function for direct transmission such that the resulting model is an optimization model. One such pair of functions is when both incidences follow the mass action law (cf. Day 2002), but a matching environmental incidence almost always exists at least locally for any incidence in direct transmission. Next, we show how the optimization model can be unfolded to yield coexistence by mutual invasibility near the singular strain. Finally, we construct a trade-off function such that evolutionary branching happens at the singular strain. This last step is known as critical function analysis. It has been shown that whenever coexistence by mutual invasibility exists near a singularity, one can choose the convexity of a trade-off function such that the singularity becomes a branching point (Bowers et al. 2005, Kisdi 2006); the technique has been applied to the evolution of pathogens e.g. by Svennungsen and Kisdi (2009), Boldin et al. (2009) and Best et al. (2010). The present paper thus expands critical function analysis by constructing incidence functions to obtain coexistence in the first place, and then continues with the existing technique to obtain evolutionary branching. The resulting branching points are structurally stable.

We illustrate the construction method by creating an example of evolutionary branching of pathogen virulence with standard incidence in direct transmission; this complements our earlier work, where we assumed mass action in direct transmission (Boldin and Kisdi in press). We assume a general trade-off structure in the sense that all pathogen-related model parameters may depend on virulence. To present a concrete example, however, we follow the common assumption that a trade-off exists between virulence and direct transmission (Anderson and May 1982), and in the last step, we perform a critical function analysis on this trade-off function.

\section{The model}

\subsection{Host-pathogen population dynamics}

We investigate the evolution of virulence in the single-infection SIR model with free pathogens 


$$
\begin{aligned}
& \frac{d S}{d t}=b-\beta f(N) \frac{I}{N} S-\gamma g(P) S-d S \\
& \frac{d I}{d t}=\beta f(N) \frac{I}{N} S+\gamma g(P) S-(d+\alpha+\rho) I \\
& \frac{d N}{d t}=b-d N-\alpha I \\
& \frac{d P}{d t}=\theta I-\sigma P
\end{aligned}
$$

where $S$ and $I$ denote respectively the population density of susceptible and infected hosts, $N$ is the total host density (the sum of $S, I$, and the density of recovered hosts), and $P$ is the density of free pathogens. The host population is regulated via a density-dependent per capita birth rate. For simplicity, we take the per capita birth rate to be inversely proportional to population density, such that hosts are born at a constant population birth rate $b$. All hosts are born susceptible, recovered hosts are immune to the pathogen, and we assume perfect cross-immunity between pathogen strains. All hosts succumb to natural death at a rate $d$. Infected hosts die due to the disease at a rate $\alpha$, the virulence, and recover at a rate $\rho$. Free pathogens are shed by infected hosts at a rate $\theta$ (there is no release of pathogens upon the host's death) and free pathogens decay at a rate $\sigma$. Upon contact with an infected host, a susceptible individual contracts the disease with probability $\beta$, whereas $\gamma$ is the infectivity of free pathogens.

The functions $f$ and $g$ describe incidence in direct and environmental transmission, respectively. $f(N)$ is thus the rate of a focal susceptible host encountering other hosts when the population density is $N$; the host thus encountered is infected with probability $I / N . g(P)$ is the rate of encountering free pathogens at pathogen density $P$. We assume that $f$ is a continuously differentiable, nondecreasing function, and construct $g$ to be also continuously differentiable and (under certain conditions) nondecreasing.

Note that the loss of free pathogens due to ingestion by a host is neglected in the last equation of (1). With biologically realistic parameter values this approximation has only a negligible effect (Dwyer 1994), and it greatly simplifies the analysis. Because our results are structurally stable, relaxing this simplification would not qualitatively change the results (although the functions constructed below would be slightly different) as long as the effect of ingestion on free pathogen density is realistically small.

Throughout this paper, we assume that (1) has an asymptotically stable equilibrium. If the model has multiple stable equilibria, we invoke the so-called Tube Theorem of Geritz et al. (2002) and restrict attention to one such equilibrium. 


\section{$2.2 \quad$ Trade-off structure}

With the exception of the birth and natural death rates of the host ( $b$ and $d$ ), all parameters in equations (1) depend on the properties of the pathogen. We assume that the pathogen strains can be identified by their virulence, $\alpha$, and this determines all other pathogen-related parameters according to twice differentiable trade-off functions. Some of these parameters, such as transmission or recovery, may of course depend also on the properties of the host, but host properties remain constant in our model. Biological considerations may suggest certain shapes for these trade-off functions, but we shall assume only that the recovery rate $\rho$ is non-increasing as a function of pathogen virulence $\left(\rho^{\prime}(\alpha) \leq 0\right)$. This assumption is made to simplify the analysis, but also appears natural as infections by more virulent strains are likely harder to clear; however we note that simple models of the host immune reaction (which do not incorporate e.g. the energetic costs inflicted by either the infection or the immune system) suggest that fast-reproducing pathogens are cleared faster (Read and Keeling 2006). We retain full flexibility with respect to all other trade-off functions, i.e., except in a few additional remarks where assumptions are explicitly stated and in the worked example of section 5 , we allow $\beta(\alpha), \gamma(\alpha), \theta(\alpha)$ and $\sigma(\alpha)$ to depend arbitrarily on virulence $\alpha$.

\subsection{Invasion fitness of a mutant strain}

Assume that a mutant strain of the pathogen appears in the resident host-pathogen system at the population dynamic equilibrium of (1). The initial dynamics of the mutant strain are given by the linearized equations

$$
\begin{aligned}
\frac{d I_{m u t}}{d t}= & \beta\left(\alpha_{m u t}\right) f(N) \frac{I_{m u t}}{N} S+\gamma\left(\alpha_{m u t}\right) g(P) \frac{P_{m u t}}{P} S \\
& -\left(d+\alpha_{m u t}+\rho\left(\alpha_{m u t}\right)\right) I_{m u t} \\
\frac{d P_{m u t}}{d t}= & \theta\left(\alpha_{m u t}\right) I_{m u t}-\sigma\left(\alpha_{m u t}\right) P_{m u t}
\end{aligned}
$$

where $S, N$ and $P$ are the equilibrium densities of the resident system (1). The leading eigenvalue associated with the linearized system (2) is positive if and only if the mutant's basic reproduction number

$$
R_{0}^{m u t}=\frac{S}{d+\alpha_{m u t}+\rho\left(\alpha_{m u t}\right)}\left[\beta\left(\alpha_{m u t}\right) \frac{f(N)}{N}+\frac{\theta\left(\alpha_{m u t}\right)}{\sigma\left(\alpha_{m u t}\right)} \gamma\left(\alpha_{m u t}\right) \frac{g(P)}{P}\right]
$$

exceeds 1 (Diekmann and Heesterbeek 2000; van den Driessche and Watmough 2002; Hurford et al. 2010; see Appendix A for derivation).

Note that the pathogen's vital rates enter $R_{0}$ only via the two functions

$$
D(\alpha)=\frac{\beta(\alpha)}{d+\alpha+\rho(\alpha)}, \quad E(\alpha)=\frac{\theta(\alpha)}{\sigma(\alpha)} \cdot \frac{\gamma(\alpha)}{d+\alpha+\rho(\alpha)}
$$


such that we have

$$
R_{0}^{m u t}\left(\alpha_{m u t}, \alpha\right)=S\left[D\left(\alpha_{m u t}\right) \frac{f(N)}{N}+E\left(\alpha_{m u t}\right) \frac{g(P)}{P}\right]
$$

(Roche et al. 2011; Boldin and Kisdi in press). $D(\alpha)$ is the number of secondary infections caused via direct transmission during the lifetime of an infection by strain $\alpha$, per unit contact rate with susceptible hosts. $D(\alpha)$ therefore measures the pathogen's adaptedness to direct transmission; similarly, $E(\alpha)$ is the pathogen's adaptedness to environmental transmission. It is important to note that $D\left(\alpha_{m u t}\right)$ and $E\left(\alpha_{m u t}\right)$ depend only on the mutant trait value $\alpha_{m u t}$ and do not depend on the resident $\alpha$. The resident influences the mutant's initial growth only via the equilibrium densities $S, N$ and $P$, which in (5) combine into two feedback variables, $S \frac{f(N)}{N}$ and $S \frac{g(P)}{P}$.

\subsection{Remarks}

If the pathogen transmits only directly $(\theta(\alpha) \equiv 0$ or $\gamma(\alpha) \equiv 0$ and therefore $E(\alpha) \equiv 0)$, then we have an optimization model with the single feedback variable $S f(N) / N$ and the optimal strain maximizes $D(\alpha)$. Similarly, if the pathogen transmits only environmentally $(\beta(\alpha) \equiv 0$ and therefore $D(\alpha) \equiv 0)$, then we have an optimization model with the single feedback $S g(P) / P$ and the optimal strain maximizes $E(\alpha)$ (see Metz et al. 2008). The feedback environment can be two dimensional only if both transmission modes are present, which is a necessary condition for evolutionary branching. In this model, evolutionary branching is driven by contrasting adaptations to the two transmission modes, i.e., by the difference between the optima of $D(\alpha)$ and $E(\alpha)$ (Boldin and Kisdi, in press).

The simplest choice of the incidence functions $f$ and $g$ is to assume mass action in both transmission modes, i.e., to assume $f(N)=N$ and $g(P)=P$. In this case, however, (5) simplifies to $R_{0}^{m u t}=S\left[D\left(\alpha_{m u t}\right)+E\left(\alpha_{m u t}\right)\right]$ such that $S$ remains the only feedback variable. Mass action therefore leads to an optimization model where the function $D(\alpha)+E(\alpha)$ is maximized (cf. Day 2002; Boldin and Kisdi in press).

\section{Preliminaries}

Evolutionary branching occurs at virulence $\alpha^{*}$ if the following conditions are satisfied (Geritz et al. 1998):

1. $\alpha^{*}$ is singular:

$$
\left[\frac{\partial R_{0}^{m u t}}{\partial \alpha_{m u t}}\right]_{\alpha_{m u t}=\alpha=\alpha^{*}}=S^{*}\left[D^{\prime}\left(\alpha^{*}\right) \frac{f\left(N^{*}\right)}{N^{*}}+E^{\prime}\left(\alpha^{*}\right) \frac{g\left(P^{*}\right)}{P^{*}}\right]=0
$$


where $S^{*}, N^{*}$ and $P^{*}$ denote the equilibrium densities in the resident population of strain $\alpha^{*}$. Note that the singularity condition requires that the signs of $D^{\prime}\left(\alpha^{*}\right)$ and $E^{\prime}\left(\alpha^{*}\right)$ are different, which means that the two transmission modes require contrasting adaptations of the pathogen.

2. $\alpha^{*}$ lacks evolutionary stability: $\mathcal{E}>0$, where

$$
\mathcal{E}=\left[\frac{\partial^{2} R_{0}^{m u t}}{\partial \alpha_{m u t}^{2}}\right]_{\alpha^{*}}=S^{*}\left[D^{\prime \prime}\left(\alpha^{*}\right) \frac{f\left(N^{*}\right)}{N^{*}}+E^{\prime \prime}\left(\alpha^{*}\right) \frac{g\left(P^{*}\right)}{P^{*}}\right]
$$

3. $\alpha^{*}$ is convergence stable: $\mathcal{E}+\mathcal{M}<0$, where

$$
\mathcal{M}=\left[\frac{\partial^{2} R_{0}^{m u t}}{\partial \alpha \partial \alpha_{m u t}}\right]_{\alpha^{*}}=S^{*}\left[\left.D^{\prime}\left(\alpha^{*}\right) \frac{d}{d \alpha} \frac{f(N)}{N}\right|_{\alpha^{*}}+\left.E^{\prime}\left(\alpha^{*}\right) \frac{d}{d \alpha} \frac{g(P)}{P}\right|_{\alpha^{*}}\right]
$$

Convergence stability and lack of evolutionary stability can hold simultaneously only if $\mathcal{M}<0$. The inequality $\mathcal{M}<0$ is the condition for mutual invasibility near the singularity (Geritz et al. 1998). Coexistence in the neighbourhood of the singularity is obviously a prerequisite for evolutionary branching, but in one-dimensional trait spaces, an evolutionary branching point automatically satisfies the condition of coexistence by mutual invasibility.

\section{The construction method}

Here we show how one can construct an example for evolutionary branching of virulence in model (1) in three steps. First, we find (locally) a specific incidence function $g_{0}$ paired to a given incidence function $f$ such that the model becomes an optimization model. Second, we unfold this optimization model by changing $g_{0}$ near the singularity to obtain a model with mutual invasibility. In the last step, we use the established technique of critical function analysis and change the curvature of a trade-off function to obtain an evolutionary branching point from a singularity with mutual invasibility.

\subsection{Constructing an optimization model}

Rewrite the basic reproduction number of a mutant strain in (5) in the form

$$
R_{0}^{m u t}=S \frac{f(N)}{N}\left[D\left(\alpha_{m u t}\right)+\frac{g(P)}{P} \frac{N}{f(N)} E\left(\alpha_{m u t}\right)\right]
$$


The mutant fitness depends on only one feedback variable, $S \frac{f(N)}{N}$, if and only if $g(P)=$ $g_{0}(P)$ with $g_{0}$ chosen such that

$$
\frac{g_{0}(P)}{P} \frac{N}{f(N)} \equiv c
$$

is a positive constant. In this case, we have an optimization model where $D(\alpha)+c E(\alpha)$ is maximized (Metz et al. 2008). The optimal virulence $\alpha^{*}$ satisfies

$$
D^{\prime}\left(\alpha^{*}\right)+c E^{\prime}\left(\alpha^{*}\right)=0, \quad D^{\prime \prime}\left(\alpha^{*}\right)+c E^{\prime \prime}\left(\alpha^{*}\right)<0
$$

which determines the location of $\alpha^{*}$ independently of the function $f$ and the still unknown function $g_{0}$.

Specify a continuously differentiable, nondecreasing function $f$ and $b, d>0$ arbitrarily. Next, specify twice differentiable trade-off functions (and hence $D$ and $E$ according to equations (4)) and $c>0$ such that the extrema of $D$ and $E$ do not coincide, an interior optimum exists according to (11), and it satisfies the non-degeneracy condition (32) given in Appendix B. Alternatively, one can specify the trade-off functions and choose $\alpha^{*}$ arbitrarily within the constraints of $(32)$ as well as that $D^{\prime}\left(\alpha^{*}\right)$ and $E^{\prime}\left(\alpha^{*}\right)$ must have opposite signs and $D^{\prime \prime}\left(\alpha^{*}\right)-\left[D^{\prime}\left(\alpha^{*}\right) / E^{\prime}\left(\alpha^{*}\right)\right] E^{\prime \prime}\left(\alpha^{*}\right)$ must be negative; then $\alpha^{*}$ is optimal with the choice $c=-D^{\prime}\left(\alpha^{*}\right) / E^{\prime}\left(\alpha^{*}\right)$.

To construct $g_{0}$ that satisfies (10), we need to solve the population dynamical equations (1) for the equilibrium densities $\hat{S}(\alpha), \hat{N}(\alpha), \hat{P}(\alpha)$ that correspond to the resident strain $\alpha$. Using (10) and after some rearrangement, the equilibrium equations are

$$
\begin{aligned}
& \hat{S}(\alpha) \frac{f(\hat{N}(\alpha))}{\hat{N}(\alpha)}[D(\alpha)+c E(\alpha)]=1 \\
& \hat{S}(\alpha)=\hat{N}(\alpha)-\left[\frac{d+\rho(\alpha)}{\alpha}\right]\left(\frac{b}{d}-\hat{N}(\alpha)\right) \\
& \hat{P}(\alpha)=\left[\frac{d}{\alpha} \frac{\theta(\alpha)}{\sigma(\alpha)}\right]\left(\frac{b}{d}-\hat{N}(\alpha)\right) \\
& \hat{I}(\alpha)=\hat{P}(\alpha) \sigma(\alpha) / \theta(\alpha)
\end{aligned}
$$

Varying $\alpha$ in some neighbourhood of $\alpha^{*}$, we solve these equations for $(\hat{N}(\alpha), \hat{P}(\alpha))$ for a range of $\alpha$ and define $g_{0}$ according to (10),

$$
g_{0}(\hat{P}(\alpha))=c \hat{P}(\alpha) \frac{f(\hat{N}(\alpha))}{\hat{N}(\alpha)}
$$

The non-degeneracy condition (32) derived in Appendix B guarantees the existence of an open interval $\left(\alpha_{\min }, \alpha_{\max }\right)$ which contains the singular virulence $\alpha^{*}$ and over which $g_{0}$ is a differentiable function. Henceforth we restrict virulence to the interval $\left(\alpha_{\min }, \alpha_{\max }\right)$ 
and perform the construction locally; one can however extend the incidence and trade-off functions in any biologically reasonable way beyond the interval $\left(\alpha_{\min }, \alpha_{\max }\right)$ without affecting the local properties (in particular, $\mathcal{E}$ and $\mathcal{M}$ ) of the singularity $\alpha^{*}$.

Biological considerations furthermore dictate that $g(P)$ should be an increasing (or at least non-decreasing) function of $P$. The necessary and sufficient conditions guaranteeing this are more stringent and also somewhat more complicated (see (37) in Appendix B). Nevertheless, for every given $f$ one can choose trade-off functions such that the resulting function $g_{0}$ is increasing. A biologically important class of incidence functions $f$ are those that satisfy $f^{\prime}\left(\hat{N}\left(\alpha^{*}\right)\right)<f\left(\hat{N}\left(\alpha^{*}\right)\right) / \hat{N}\left(\alpha^{*}\right)$; this holds for standard incidence (constant $f$ ) and whenever $f$ is concave with $f(0) \geq 0$. For this class of incidence functions, and assuming realistically that $\theta$ is increasing and $\sigma$ is non-increasing, any convex or linear function $\theta$ with $\theta(0)=0$ leads to an increasing function $g_{0}$ (this however is not necessary for $g_{0}$ to be increasing; see Appendix B).

With $g(P)=g_{0}(P)$, the model is an optimization model over the interval $\left(\alpha_{\min }, \alpha_{\max }\right)$ with locally optimal virulence $\alpha^{*}$. This implies that no pair of strains may exhibit either mutual invasibility or mutual exclusion, and therefore $\mathcal{M}=0$ at $\alpha^{*}$. In the next step, we perturb the incidence function $g_{0}$ to obtain a model with $\mathcal{M}<0$ and therefore with mutual invasibility near $\alpha^{*}$.

\subsection{Unfolding the optimization model}

An important observation is that the singularity condition (6) and $\mathcal{M}$ in (8) depend only on the values and first derivatives of $f$ and $g$ at the equilibrium population densities of the singular strain $\left(S^{*}=\hat{S}\left(\alpha^{*}\right), N^{*}=\hat{N}\left(\alpha^{*}\right), P^{*}=\hat{P}\left(\alpha^{*}\right)\right)$, which themselves depend only on the values of $f$ and $g$. Hence one can take any function $g$ tangent to $g_{0}$ at the singularity $\left(g\left(P^{*}\right)=g_{0}\left(P^{*}\right)\right.$ and $\left.g^{\prime}\left(P^{*}\right)=g_{0}^{\prime}\left(P^{*}\right)\right)$ in place of $g_{0}$ and still have a singularity at $\alpha^{*}$ with $\mathcal{M}=0$. Note that this perturbed model is no longer an optimization model and it can accommodate either mutual invasibility or mutual exclusion between strains. However, near the singularity the area of mutual invasibility or mutual exclusion is cusp-shaped such that, corresponding to $\mathcal{M}=0$, it vanishes in first order (see Figure 2 below; Dercole and Geritz in prep.).

Changing the derivative $g^{\prime}\left(P^{*}\right)$ while retaining the value $g\left(P^{*}\right)$ changes $\mathcal{M}$ but does not change the location of the singularity $\alpha^{*}$. In Appendix B, we derive the transversality condition for unfolding $\mathcal{M}=0$ and show that it is always satisfied, i.e., $\mathcal{M}$ changes sign when $g^{\prime}\left(P^{*}\right)$ is varied across $g_{0}^{\prime}\left(P^{*}\right)$, whenever the non-degeneracy condition (32) holds and therefore $g_{0}^{\prime}\left(P^{*}\right)$ exists. We can thus always obtain a singularity with $\mathcal{M}<0$ by changing the slope of $g$ locally. The derivative of $\mathcal{M}$ with respect to the slope of $g$ is given

in formula (49) of Appendix B. To obtain $\mathcal{M}<0$, the slope of $g$ should be increased (decreased) relative to the slope of $g_{0}$ at $P^{*}$ if (49) is negative (positive). $\mathcal{M}<0$ im- 
plies mutual invasibility near the singularity and is a necessary condition for evolutionary branching.

\subsection{Obtaining an evolutionary branching point}

The last step follows earlier work by de Mazancourt and Dieckmann (2004), Bowers et al. (2005) and Kisdi (2006); the method used in this section has been applied to various other models by Geritz et al. (2007), Svennungsen and Kisdi (2009), Boldin et al. (2009), Best et al. (2010) and others.

Recall that evolutionary branching occurs at $\alpha^{*}$ if $\mathcal{E}>0$ and $\mathcal{E}+\mathcal{M}<0$. We thus need to tune the model to have $\mathcal{E}$ in the interval $(0,-\mathcal{M})$, while keeping the location of the singularity $\alpha^{*}$ fixed and $\mathcal{M}<0$ as obtained above. The values of $\frac{f\left(N^{*}\right)}{N^{*}}$ and $\frac{g\left(P^{*}\right)}{P^{*}}$ are now fixed and $\mathcal{E}$ does not depend on the derivatives of the incidence functions; hence to obtain a desired value of $\mathcal{E}$, we need to modify a trade-off function appropriately.

The key observation in this step is that whereas $\alpha^{*}$ and $\mathcal{M}$ depend only on the first derivatives of the trade-off functions (via $D^{\prime}$ and $E^{\prime}$ in (6) and in (8), respectively), $\mathcal{E}$ depends on the second derivatives (cf. (7)). Hence by changing the convexity of a tradeoff function at $\alpha^{*}$ without changing its value and its first derivative at the same point, we can change $\mathcal{E}$ arbitrarily without changing $\alpha^{*}$ or $\mathcal{M}$. In particular, we can choose the convexity of a trade-off such that $\mathcal{E}>0$ and $\mathcal{E}+\mathcal{M}<0$ are satisfied, and therefore $\alpha^{*}$ is an evolutionary branching point.

\section{Example: Standard incidence in direct transmis- sion}

In this section, we illustrate the above methods by investigating the evolution of virulence assuming standard incidence in direct transmission. In many instances, the rate of encounters does not follow the common assumption of mass action, i.e., is not proportional to population density, because hosts actively seek out conspecifics while they search for mates or engage in other social interactions. Standard incidence assumes that hosts encounter other hosts at a fixed, density-independent rate, which can be seen as an extreme case of seeking contacts actively. (An alternative interpretation of standard incidence is to assume that the area occupied by the population changes proportionally to the number of individuals such that population density is constant, and the rate of encounters is proportional to density as in mass action. The model in (1) however assumes a constant area by treating population size and density interchangeably, and therefore we do not follow this alternative interpretation.) 
Under standard incidence, $f(N) \equiv f_{0}$. To avoid excess notation, we factor the constant $f_{0}$ into $\beta$, and henceforth assume $f(N)=1$. Note that with $f_{0}$ factored in, $\beta$ is no longer a probability; it is now measured in units of $1 /$ time and need not be less than 1 .

For the example we work out, we assume $b=1$ and $d=0.1$ for the host life history parameters and $\beta(\alpha)=\frac{20 \alpha}{1+\alpha}, \theta(\alpha)=\frac{5 \alpha}{5+\alpha}, \gamma(\alpha) \equiv \gamma_{0}=1, \rho(\alpha) \equiv \rho_{0}=1, \sigma(\alpha) \equiv \sigma_{0}=0.1$ for the pathogen's trade-offs (note that we shall change the function $\beta$ in the last step of constructing a model with evolutionary branching). Moreover, we choose $c=1$. By equation (11), these choices imply that the singular virulence is at $\alpha^{*}=1.5146$.

We start with solving equations (12) for the equilibrium densities of a given resident strain $\alpha$. Because $f(N)=1$, the first two of equations of (12) yield a linear equation for $\hat{N}(\alpha)$, which is readily solved; the other densities follow immediately. The equilibrium densities are positive for $0.039<\alpha<65.027$.

In Figure 1a, we plot the parametric curve between $\hat{P}(\alpha)$ and $c \hat{P}(\alpha) / \hat{N}(\alpha)$ (cf. (13)). To obtain the function $g_{0}$ locally, we restrict virulence to the interval $\left(\alpha_{\min }, \alpha_{\max }\right)=(1,2)$. This interval contains the singularity at $\alpha^{*}=1.5146$; moreover, $g_{0}(P)$ as defined by $g_{0}(\hat{P}(\alpha))=c \hat{P}(\alpha) / \hat{N}(\alpha)$ is an increasing function of $P$ in the interval $3.716<P<4.410$, which is the range of $\hat{P}(\alpha)$ over $\alpha \in(1,2)$ (cf. Figure 1a). The population dynamics of the singular strain $\alpha^{*}$ in $(1)$ with $g(P)=g_{0}(P)$ have a locally asymptotically stable equilibrium. By continuity, the equilibrium is stable also in an open interval around $\alpha^{*}$ and also under small changes in the incidence function $g$.

With the choice $g(P)=g_{0}(P)$, the model is an optimization model and therefore the singularity is characterized with $\mathcal{M}=0$. Figure $1 \mathrm{~b}$ shows the corresponding mutual invasibility plot, i.e., the pairwise invasibility plot superimposed with its mirror image (Geritz et al. 1998). The pairwise invasibility plot is the sign plot of $\ln R_{0}^{m u t}\left(\alpha_{2}, \alpha_{1}\right)$ obtained from the basic reproduction number in (5), which shows whether strain $\alpha_{2}$ is able to invade the resident population of $\alpha_{1}$ (shown by the first sign in the labels); whereas its mirror image is the sign plot of $\ln R_{0}^{m u t}\left(\alpha_{1}, \alpha_{2}\right)$, which indicates invasion under the reversal of roles (second sign in the labels). The pairwise invasibility plot in Figure 1b is skew-symmetric (positive areas mirror into negative and vice versa). This follows directly from the model being an optimization model and indicates that neither mutual invasibility nor mutual exclusion occurs for any pair of strains (see Metz et al. (2008) and Gyllenberg and Service (2011) for further details on pairwise invasibility plots of optimization models).

We unfold the above optimization model first such that the singularity still retains $\mathcal{M}=0$. To this end, we choose a new function $g_{1}$ that is tangential to $g_{0}$ at $P^{*}=\hat{P}\left(\alpha^{*}\right)$. The simplest choice is the linear function

$$
g_{1}(P)=g_{0}\left(P^{*}\right)+g_{0}^{\prime}\left(P^{*}\right)\left(P-P^{*}\right)
$$

but this function gives negative incidences at low densities of free pathogens (see Figure 
(b)

(a)
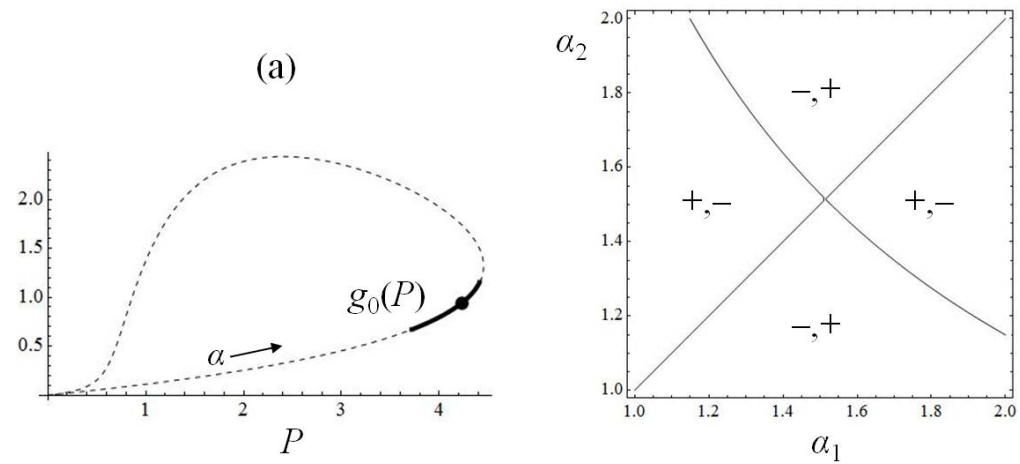

Figure 1: (a) The dashed line is the parametric curve $(\hat{P}(\alpha), c \hat{P}(\alpha) / \hat{N}(\alpha))$ with $\alpha$ varied over the full interval of pathogen viability; the arrow indicates the direction of increasing $\alpha$. The thick part shows the restriction to $\alpha \in\left(\alpha_{\min }, \alpha_{\max }\right)=(1,2)$ that we use for the function $g_{0}$ and the dot marks the position of the singularity. (b) The pairwise invasibility plot and its mirror image superimposed for $g(P)=g_{0}(P)$. In the areas marked with " $+,-, ", \alpha_{2}$ invades $\alpha_{1}$ whereas $\alpha_{1}$ does not invade $\alpha_{2}$; in the areas marked with "-, + ", the opposite happens. Since with $g(P)=g_{0}(P)$ the model is an optimization model, no other sign combinations appear.

2a); although it can be truncated at zero with no consequence to the properties of the singularity, the resulting incidence function is still perhaps not realistic. We shall therefore use the function

$$
g_{1}(P)=\frac{P^{3}}{P^{* 3}}\left[g_{0}\left(P^{*}\right)+\left(g_{0}^{\prime}\left(P^{*}\right)-3 \frac{g_{0}\left(P^{*}\right)}{P^{*}}\right)\left(P-P^{*}\right)\right]
$$

which is also tangential to $g_{0}$ at $P^{*}$ (Figure $\left.2 \mathrm{a}\right)$.

Because $\mathcal{M}$ depends only on the value and the first derivative of the incidence function $g, \mathcal{M}$ is zero with the choice $g(P)=g_{1}(P)$ and therefore the invasion boundaries in Figure $2 \mathrm{~b}$ coincide up to first order at the singularity. The model is however no longer an optimization model; there is a cusp-shaped area of mutual exclusion, where neither strain can invade the population of the other.

In order to obtain a model with $\mathcal{M}<0$ and hence with mutual invasibility, we need to change the slope of the incidence function $g$ at the singularity. The derivative of $\mathcal{M}$ with respect to the slope of $g$ is given by expression (49) in Appendix B, which in the present model evaluates to 0.3276 . Therefore, we decrease the derivative by changing the incidence function in (15) into

$$
g_{2}(P)=\frac{P^{3}}{P^{* 3}}\left[g_{0}\left(P^{*}\right)+\left(\left(g_{0}^{\prime}\left(P^{*}\right)-\Delta\right)-3 \frac{g_{0}\left(P^{*}\right)}{P^{*}}\right)\left(P-P^{*}\right)\right]
$$


(b)
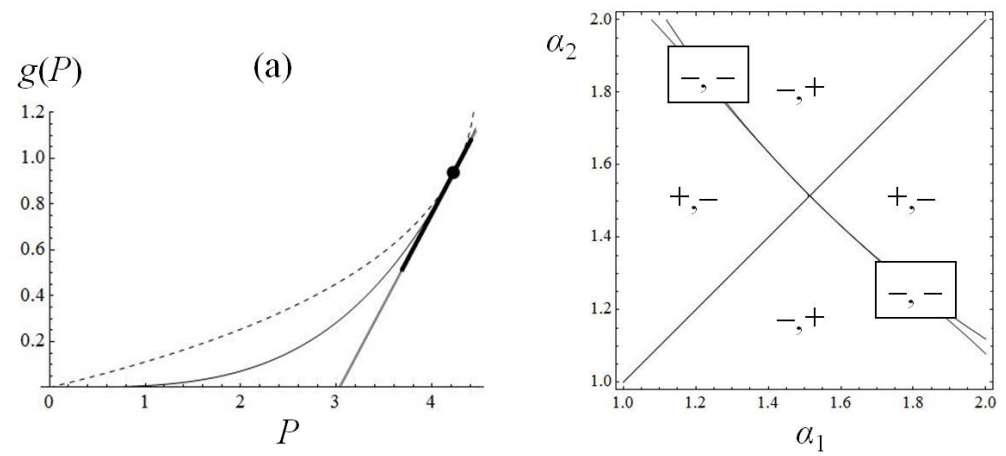

Figure 2: (a) The grey and black lines show $g_{1}$ according to (14) and (15), respectively; both are tangential to the parametric curve in Figure 1 (dashed line) at the singularity. The thick black line indicates the segment used to obtain the mutual invasibility plot. (b) The mutual invasibility plot for $g(P)=g_{1}(P)$ as given by the linear function in (14). Note the cusp-shaped area of mutual exclusion ("-,-"). The function in (15) yields a similar plot but with less separation between the boundaries.

with some positive but sufficiently small $\Delta$. The resulting function is shown in Figure 3a. In the corresponding mutual invasibility plot (Figure $3 \mathrm{~b}$ ), the invasion boundaries separate also in first order and thus form a wedge-shaped area of coexistence by mutual invasibility.

Notice that in the first step of unfolding we got a cusp-shaped area of mutual exclusion (Figure 2b), whereas in the present step there is a wedge-shaped area of mutual invasibility (Figure 3b). Figure 4 depicts schematically how this change happens. As $\mathcal{M}$ decreases from zero (left panel in Figure 4) to slightly negative (middle panel), the curved invasion boundaries move from tangential to intersecting, such that near the singularity there appears an area of mutual invasibility, but further away there is still an area of mutual exclusion. As $\mathcal{M}$ becomes increasingly negative, the point of intersection moves away from the singularity and therefore the area of mutual exclusion disappears. Priklopil (2012) proved that the scenario depicted in Figure 4 is one of the two generic scenarios that can occur when $\mathcal{M}$ changes sign (in the other scenario, the invasion boundaries intersect while $\mathcal{M}$ is positive). Moreover, Priklopil (2012) also shoved that in the neighbourhood of the intersection point of the invasion boundaries, there is an area of unprotected coexistence, i.e., there exist pairs of strains outside the area of mutual invasibility that can coexist. The extension of equations (1) to two resident strains of the pathogen thus has multiple attractors of population dynamics for some choices of the incidence functions.

In the last step of the construction, we change the trade-off function $\beta$ into a new function $\beta_{B P}$ that is tangential to $\beta$ at the singularity (the choice of $\beta$ is arbitrary, one could change any of the trade-off functions analogously). Because $\beta_{B P}\left(\alpha^{*}\right)=\beta\left(\alpha^{*}\right)$ and $\beta_{B P}^{\prime}\left(\alpha^{*}\right)=\beta^{\prime}\left(\alpha^{*}\right)$, this change does not affect the position of the singularity or 
(b)
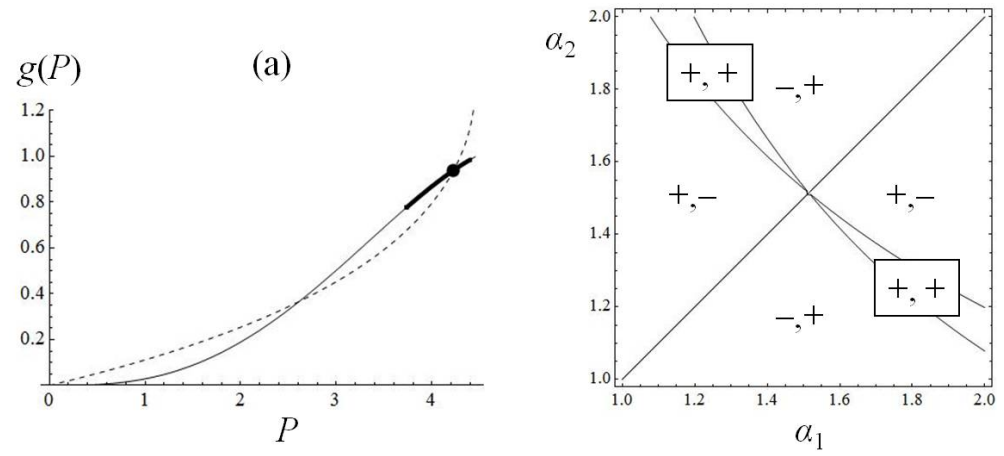

Figure 3: (a) The black line shows $g_{2}$ according to (16) with $\Delta=0.5$; the thick part is used to obtain the mutual invasibility plot. At the singularity, $g_{2}$ has the same value but a smaller derivative compared to the parametric curve in Figure 1 (dashed line). (b) The mutual invasibility plot for $g(P)=g_{2}(P)$ exhibits a wedge-shaped area of coexistence by mutual invasibility $("+,+")$.

(a)

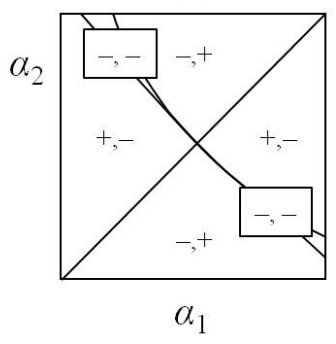

(b)

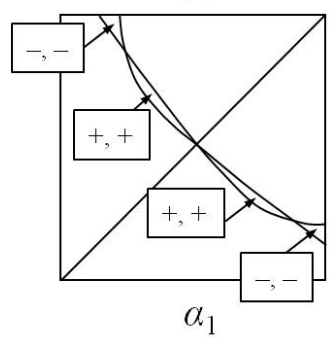

(c)

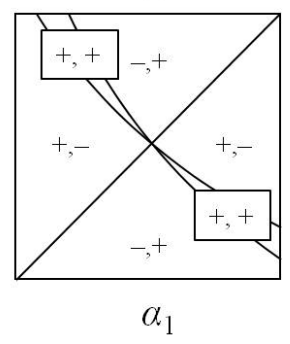

Figure 4: A schematic visualization of how mutual exclusion is replaced with mutual invasibility (the curvature of the invasion boundaries is exaggerated for clarity). (a) $\mathcal{M}=0$, a cusp-shaped area of mutual exclusion is connected to the singularity as in Figure 2b; (b) $\mathcal{M}$ is small negative, a wedge-shaped area of mutual invasibility connects to the singularity and the area of mutual exclusion shrinks away; (c) $\mathcal{M}$ is more strongly negative, the area of mutual exclusion has disappeared as in Figure $3 \mathrm{~b}$. 
the value of $\mathcal{M}$ (cf. (6) and (8)). We continue to use the incidence function $g_{2}$ in (16) with $\Delta=0.5$, which implies $\mathcal{M}=-0.01915$. In order to obtain an evolutionary branching point, the convexity $\beta_{B P}^{\prime \prime}\left(\alpha^{*}\right)$ must be chosen such that $\mathcal{E}$ in $(7)$ is in the interval $(0,-\mathcal{M})=(0,0.01915)$; with the trade-off and incidence functions of this example, this occurs for $1.8084<\beta_{B P}^{\prime \prime}\left(\alpha^{*}\right)<2.2618$. We therefore choose $\beta_{B P}^{\prime \prime}\left(\alpha^{*}\right)=2$ and use the sigmoidal function

$$
\beta_{B P}(\alpha)=A+\frac{B}{1+C \exp (-k \alpha)}
$$

with $k=1.2$ and $A, B, C$ chosen such that $\beta_{B P}$ is tangential to $\beta$ at $\alpha^{*}$ and has the required convexity (Figure 5a).

In Figure 5b we show the pairwise invasibility plot obtained with the constructed incidence function $g_{2}$ and trade-off function $\beta_{B P}$. As prescribed by the construction, the model now exhibits an evolutionary branching point at $\alpha^{*}$.

(b)
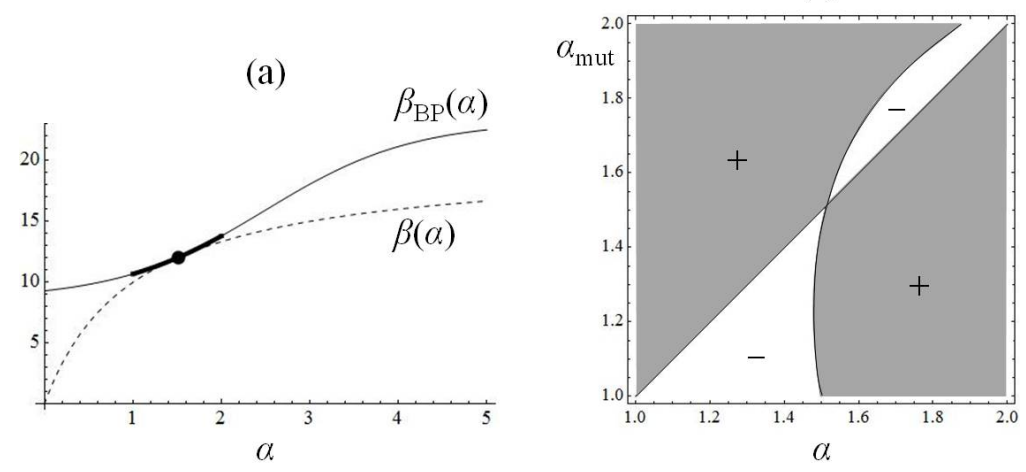

Figure 5: (a) The original trade-off function $\beta$ (dashed line) and $\beta_{B P}$ given in (17) with parameters $A=8.5943, B=14.5957, C=19.8738$ (black line; the thick part shows the part used to obtain the pairwise invasibility plot). (b) The pairiwise invasibility plot with the incidence function $g(P)=g_{2}(P)$ given by (16) with $\Delta=0.5$ and trade-off function $\beta_{B P}$. In the grey areas strain $\alpha_{m u t}$ can invade strain $\alpha$; the singularity is an evolutionary branching point.

\section{Discussion}

In this paper, we introduced a constructive method to find incidence functions that allow for coexistence by mutual invasibility of strains near an evolutionary singularity. Once such coexistence is possible, critical function analysis can be used to obtain a trade-off function that leads to evolutionary branching (Bowers et al. 2005, Kisdi 2006). Evolutionary branching is structurally stable, i.e., it does not depend on the exact particulars 
of the constructed example but holds for similar incidence and trade-off functions as well. Moreover, only the local properties of these functions matter (up to the first order for the incidence function and second order for the trade-off function), beyond which the constructed functions can be chosen arbitrarily without any change to the evolutionary singularity. This mathematical flexibility helps to construct examples with biologically realistic functions. However, the construction method may also indicate that no biologically realistic function yields the requested outcome; this would happen for example if it turned out that branching at a given singularity would be possible only with a decreasing incidence function.

Most models of evolutionary ecology assume a number of functions without firm justifications, and it is very difficult to judge to which extent these choices influence the model predictions. Boldin et al. (2009) found that the conditions for evolutionary branching of pathogens depend on the shape of the function that describes the host's density-dependent birth rate; this is worrisome because the host population dynamics is commonly assumed on an ad hoc basis. In our present model, there are two incidence functions and five trade-off functions. Deciding whether this model can lead to evolutionary branching would be very difficult if one simply tries various incidence and trade-off functions, as in a random search there is no guarantee for success and failure does not prove non-existence.

For some of the simplest choices of functions, evolutionary branching is indeed impossible. This is the case if both incidence functions follow mass action, which results in an optimization model (Day 2002; Boldin and Kisdi in press). An optimization model however needs to be changed only slightly in order to obtain an evolutionary branching point; as Boldin and Kisdi (in press) show, it suffices to assume a concave rather than a linear function for the environmental incidence function when the direct transmission follows mass action. This is also the basis of our present construction method. If an optimization model can be constructed by the appropriate choice of an incidence function, then a straightforward unfolding will yield coexistence and evolutionary branching.

There is an unexpected upshot of our construction method. As we unfold a model with $\mathcal{M}=0$ into a model with nonzero $\mathcal{M}$, we encounter a situation where the invasion boundaries generically intersect each other (cf. Figure 4); and in the neighbourhood of such an intersection point, coexistence occurs also outside the area of mutual invasibility (Priklopil 2012). Hence the construction method yields not only examples for evolutionary branching, but also examples of unprotected coexistence with multiple attractors in the population dynamics. Most well-known simple models of disease dynamics lack multiple attractors, but our analysis suggests that with more general choices of the model's feedback functions and with multiple strains of the pathogen, multiple attractors appear naturally.

The incidence functions are important in the adaptive dynamics of the evolving pathogen because they determine the feedback loop through which the pathogens influence each oth- 
ers' reproduction (cf. (5)). The feedback loop has a central role in population dynamics (Diekmann et al. 2001, 2003; Meszéna et al. 2006) and epidemiological dynamics (Andreasen and Pugliese 1995) as well as in evolution (Mylius and Diekmann 1995). In most evolutionary models, however, it is only the dimensionality of the feedback loop that has received proper attention but not the shape of the feedback functions (see e.g. Pugliese 2002; Dieckmann and Metz 2006; Metz et al. 2008; Svennungsen and Kisdi 2009; Best et al. 2010). A minimum of two feedback variables may lead to either mutual invasibility or mutual exclusion, but these two possibilities allow for very different evolutionary scenarios. Whether the model (with a given set of trade-off functions) exhibits mutual invasibility rather than mutual exclusion is determined by the shape of the feedback functions, and this is what our analysis focuses on.

The construction method we used to study the evolutionary dynamics of pathogen virulence is applicable to a wide range of other evolutionary models as well. The key point is to find feedback functions such that mutual invasibility occurs near a singularity. The position of the singularity remains fixed as long as the values of the feedback functions remain unchanged (cf. (6)). $\mathcal{M}$ however depends also on the slopes (but not on higher derivatives) of the feedback functions; this is because the feedback variables depend only on the resident trait value and in $\mathcal{M}$ the invasion fitness is differentiated only once with respect to the resident (cf. (8)). By changing the slopes of the feedback functions but not their values, one can change $\mathcal{M}$ such that the position of the singularity remains fixed. If one chooses the slopes such that $\mathcal{M}<0$, then the condition for mutual invasibility near the fixed singularity is satisfied. The most straightforward way of finding feedback functions that yield $\mathcal{M}<0$ is to construct a set of functions that yield $\mathcal{M}=0$ and unfold this degeneracy. Mutual invasibility near the singularity makes evolutionary branching possible when the convexity of a trade-off can be chosen appropriately (Bowers et al. 2005, Kisdi 2006).

When the incidence and trade-off functions are specified a priori, their values and derivatives can be changed by changing the parameters that appear in those functions. The values and derivatives at given points are however linked via the specified functional forms, which constrains the possible outcomes of the model. Our construction method effectively treats the values and derivatives of the incidence and trade-off functions directly as model parameters. In this paper, we focused only on constructing examples for evolutionary branching; but the approach can be extended to characterize all possible functions that lead to a certain evolutionary outcome (such as evolutionary branching) in terms of relatively few parameters. This can then be compared with biologically realistic shapes of functions, obtained from measurements in various experimental systems or by modelling the biological processes underlying the trade-offs (e.g. Gilchrist and Sasaki 2002; Alizon and van Baalen 2005; Read and Keeling 2006; Boldin and Diekmann 2008; see Mideo et al. 2008 for a review) and incidence functions (Breban et al. 2010). In other words, the adaptive dynamics need not be re-investigated for each case study proposing different incidence and trade-off functions. Instead, a powerful way of analysis is to derive conditions 
for certain evolutionary outcomes without sacrificing generality with ad hoc assumptions about the functions involved in the model, and then compare these conditions with the actual functions obtained in various concrete systems.

\section{Acknowledgements}

We thank two anonymous referees for their helpful comments. E.K. was financially supported by the Academy of Finland and B.B. was supported by the Slovenian Research Agency (project no. Z7-3658 and BI-FI/12-13-010).

\section{References}

[1] Alizon S. and van Baalen M. 2005. Emergence of a convex trade-off between transmission and virulence. Am. Nat. 165: E155-E167.

[2] Anderson R. M. and May R. M. 1982. Coevolution of hosts and parasites. Parasitology 85: $411-426$.

[3] Andreasen V. and Pugliese A. 1995. Pathogen coexistence induced by densitydependent host mortality. J. Theor. Biol. 177: 159-165.

[4] Best A., White A., Kisdi E., Antonovics J., Brockhurst M. and Boots M. 2010. The evolution of host-parasite range. Am. Nat. 176: 63-71.

[5] Boldin B. and Diekmann O. 2008. Superinfections can induce evolutionarily stable coexistence of pathogens. J. Math. Biol. 56: 635-672.

[6] Boldin B., Geritz S. A. H. and Kisdi E. 2009. Superinfections and adaptive dynamics of pathogen virulence revisited: a critical function analysis. Evol. Ecol. Res. 11: 153-175.

[7] Boldin B. and Kisdi E. On the evolutionary dynamics of pathogens with direct and environmental transmission. Evolution (in press, DOI: 10.1111/j.1558-5646.2012.01613.x)

[8] Bowers R. G., Hoyle A., White A. and Boots M. 2005. The geometric theory of adaptive evolution: Trade-off and invasion plots. J. Theor. Biol. 233, 363-377.

[9] Breban R. Role of environmental persistence in pathogen transmission: a mathematical approach. J. Math. Biol. (in press, DOI 10.1007/s00285-012-0520-2)

[10] Breban R., Drake J. M. and Rohani P. 2010. A general multi-strain model with environmental transmission: invasion conditions for the disease-free and endemic states. J. theor. Biol. 264: 729-736.

[11] Colwell, R. R., and A. Huq. 1994. Environmental reservoir of Vibrio cholerae. The causative agent of cholera. Ann. N. Y. Acad. Sci. 740: 44-54. 
[12] Day T. 2002. Virulence evolution via host exploitation and toxin production in sporeproducing pathogens. Ecol. Letters 5: 471-476.

[13] de Jong M. C. M., Diekmann O. and Heesterbeek H. 1995. How does transmission of infection depend on population size? In: Denis Mollison (ed): Epidemic models: Their structure and relation to data. Cambridge University Press, pp. 84-94.

[14] de Mazancourt C. and Dieckmann U. 2004. Trade-off geometries and frequencydependent selection. Am. Nat. 164: 765-778.

[15] Dercole F. and Geritz S. A. H. Unfolding the resident-invader dynamics of similar strategies (in preparation)

[16] Dieckmann U. and Metz J. A. J. 2006. Surprising evolutionary predictions from enhanced ecological realism. Theor. Pop. Biol. 69: 263-281.

[17] Diekmann O., Gyllenberg M., Huang H., Kirkilionis M., Metz J. A. J. and Thieme H. R. 2001. On the formulation and analysis of general deterministic structured population models. II. Nonlinear theory. J. Math. Biol. 43: 157-189.

[18] Diekmann O., Gyllenberg M. and Metz J. A. J. 2003. Steady state analysis of structured population models. Theor. Pop. Biol. 63: 309-338.

[19] Diekmann O. and Heesterbeek J. A. P. 2000. Mathematical epidemiology of infectious diseases: Model building, analysis and interpretation. John Wiley and Sons Inc., Chichester, UK.

[20] Dwyer G. 1994. Density dependence and spatial structure in the dynamics of insect pathogens. Am. Nat. 143: 533-562.

[21] Geritz S. A. H., Gyllenberg M., Jacobs F. and Parvinen K. 2002. Invasion dynamics and attractor inheritance. J. Math. Biol. 44: 548-560.

[22] Geritz S. A. H. Kisdi E., Meszéna G. and Metz J.A.J. 1998. Evolutionarily singular strategies and the adaptive growth and branching of the evolutionary tree. Evol. Ecol. 12: $35-57$.

[23] Geritz S. A. H. Kisdi E. and Yan P. 2007. Evolutionary branching and long-term coexistence of cycling predators: Critical function analysis. Theor. Pop. Biol. 71: 424435.

[24] Gilchrist M. A. and Sasaki A. 2002. Modeling host-parasite coevolution: A nested approach based on mechanistic models. J. Theor. Biol. 218: 289-308.

[25] Gyllenberg M. and Service R. 2011. Necessary and sufficient conditions for the existence of an optimisation principle in evolution. J. Math. Biol. 62: 359-369. 
[26] Hunford A., Cownden D. and Day T. 2010. Next-generation tools for evolutionary invasion analyses. J. R. Soc. Interface 7: 561-571.

[27] King A. A., Ionides E. L., Pascual M. and Bouma M. J. 2008. Inapparent infections and cholera dynamics. Nature 454: 877-880.

[28] Kisdi E. 2006. Trade-off geometries and the adaptive dynamics of two co-evolving species. Evol. Ecol. Res. 8: 959-973.

[29] Li S., Eisenberg J., Spicknall I., Koopman J. 2009 Dynamics and control of infections transmitted from person to person through the environment. Am. J. Epidemiol. 170:257265.

[30] Meszéna G., Gyllenberg M., Psztor L. and Metz J. A. J. 2006. Competitive exclusion and limiting similarity: A unified theory. Theor. Pop. Biol. 69: 68-87.

[31] Metz J. A. J., Mylius S. and Diekmann O. 2008. When does evolution optimize? Evol. Ecol. Res. 10: 629-654.

[32] Mideo N., Alizon S. and Day T. 2008. Linking within- and between-host dynamics in the evolutionary epidemiology of infectious diseases. Trends Ecol. Evol. 23: 511-517.

[33] Mylius S. D. and Diekmann O. 1995. On evolutionarily stable life histories, optimization and the need to be specific about density dependence. Oikos 74: 218-224.

[34] Pugliese A. 2002. On the evolutionary coexistence of parasite strains. Math. Biosci. 177-178: $355-375$.

[35] Priklopil T. 2012. On invasion boundaries and the unprotected coexistence of two strategies. J. Math. Biol. 64: 1137-1156.

[36] Roche B., Drake J. and Rohani P. 2011. The curse of the pharaoh revisited: Evolutionary bi-stability in environmentally transmitted pathogens. Ecol. Letters 14: 569575.

[37] Read J. M. and M. J. Keeling. 2006. Disease evolution across a range of spatiotemporal scales. Theor. Pop. Biol. 70: 201-213.

[38] Rohani P., Breban R., Stallknecht D. E. and Drake, J. M. 2009. Environmental transmission of avian influenza viruses and its implications for disease control. Proc. Natl. Acad. Sci. U.S.A. 106: 10365-10369.

[39] Svennungsen T. and Kisdi E. 2009. Evolutionary branching of virulence in a singleinfection model. J. theor. Biol. 257: 408-418.

[40] Van den Driessche P. and Watmough J. 2002. Reproduction numbers and subthreshold endemic equilibria for compartmental models of disease transmission. Math. Biosci. 180: 29-48. 
[41] Walther B. A. and Ewald P. W. 2004. Pathogen survival in the external environment and the evolution of virulence. Biol. Rev. 79: 849-869.

\section{Appendix A}

In this Appendix, we derive the basic reproduction number of a mutant strain, $R_{0}^{m u t}$. The basic reproduction number is the number of new infections made by an infected host during the entire lifetime of the infection via either transmission modes, i.e., the leading eigenvalue of the next-generation matrix (Diekmann and Heesterbeek, 2000).

The mutant's dynamics in (2) can be written as

$$
\left(\begin{array}{c}
\dot{I}_{\text {mut }} \\
\dot{P}_{m u t}
\end{array}\right)=\left(\begin{array}{cc}
\beta_{m u t} \frac{f(N)}{N} S-\left(d+\alpha_{m u t}+\rho_{m u t}\right) & \gamma_{m u t} \frac{g(P)}{P} S \\
\theta_{\text {mut }} & -\sigma_{m u t}
\end{array}\right)\left(\begin{array}{c}
I_{m u t} \\
P_{m u t}
\end{array}\right)
$$

where the shorthand $\beta_{\text {mut }}=\beta\left(\alpha_{\text {mut }}\right)$ etc. is used for brevity. To obtain the nextgeneration matrix, we write the matrix in (18) as $\mathbf{F}+\mathbf{\Sigma}-\mathbf{V}$, where

$$
\mathbf{F}=\left(\begin{array}{cc}
\beta_{m u t} \frac{f(N)}{N} S & \gamma_{m u t} \frac{g(P)}{P} S \\
0 & 0
\end{array}\right)
$$

is the reproduction matrix,

$$
\boldsymbol{\Sigma}=\left(\begin{array}{cc}
0 & 0 \\
\theta_{\text {mut }} & 0
\end{array}\right)
$$

is the transition matrix and

$$
\mathbf{V}=\left(\begin{array}{cc}
d+\alpha_{m u t}+\rho_{m u t} & 0 \\
0 & \sigma_{m u t}
\end{array}\right)
$$

is the matrix of death rates. The next-generation matrix is then given by

$$
\mathbf{F}(\mathbf{V}-\boldsymbol{\Sigma})^{-1}=\left(\begin{array}{cc}
\frac{S}{d+\alpha_{m u t}+\rho_{m u t}}\left[\beta_{m u t} \frac{f(N)}{N}+\frac{\theta_{m u t}}{\sigma_{m u t}} \gamma_{m u t} \frac{g(P)}{P}\right] & \frac{\gamma_{m u t}}{\sigma_{m u t}} \frac{g(P)}{P} S \\
0 & 0
\end{array}\right)
$$

(Diekmann and Heesterbeek, 2000). This matrix is of rank 1 and its leading eigenvalue is its first row, first column element, which gives $R_{0}^{m u t}$ in (3).

The next-generation theorem (Diekmann and Heesterbeek 2000, theorem 6.13; van den Driessche and Watmough 2002, theorem 2; Hurford et al. 2010) states that the trivial equilibrium of (18) is stable (unstable) if $R_{0}^{\text {mut }}<1\left(R_{0}^{\text {mut }}>1\right)$, provided that $\mathbf{F}$ and $(\mathbf{V}-\boldsymbol{\Sigma})^{-1}$ are nonnegative matrices and all eigenvalues of $(\mathbf{V}-\mathbf{\Sigma})$ are positive. It is straightforward to see that these conditions are satisfied; $\mathbf{F}$ and

$$
(\mathbf{V}-\boldsymbol{\Sigma})^{-1}=\left(\begin{array}{cc}
\frac{1}{d+\alpha_{m u t}+\rho_{m u t}} & 0 \\
\frac{\theta_{m u t}}{\sigma_{m u t}} \frac{1}{d+\alpha_{m u t}+\rho_{m u t}} & \frac{1}{\sigma_{m u t}}
\end{array}\right)
$$


are indeed nonnegative, and the eigenvalues of the triangular matrix

$$
\mathbf{V}-\boldsymbol{\Sigma}=\left(\begin{array}{cc}
d+\alpha_{m u t}+\rho_{m u t} & 0 \\
-\theta_{\text {mut }} & \sigma_{m u t}
\end{array}\right)
$$

are its diagonal elements, which are both positive. Hence the spectral bound of (2) is positive if and only if $R_{0}^{\text {mut }}$ in (3) exceeds 1 .

\section{Appendix B}

Here we derive non-degeneracy and transversality conditions guaranteeing respectively (i) that there is an interval $\left(\alpha_{\min }, \alpha_{\max }\right)$ which contains the singularity $\alpha^{*}$ and over which $g_{0}(\hat{P}(\alpha))$ defined in (13) is an (increasing) function; and (ii) that changing the derivative of $g$ at the singularity relative to that of $g_{0}$ changes the cross derivative $\mathcal{M}$ in (8) from zero to negative.

\subsection{Preliminaries}

The equilibrium population densities of a resident population harbouring an arbitrary strain $\alpha$ are given by the equations

$$
\begin{aligned}
& \hat{S}(\alpha)\left[D(\alpha) \frac{f(\hat{N}(\alpha))}{\hat{N}(\alpha)}+E(\alpha) \frac{g(\hat{P}(\alpha))}{\hat{P}(\alpha)}\right]=1 \\
& \hat{S}(\alpha)=\hat{N}(\alpha)-\left[\frac{d+\rho(\alpha)}{\alpha}\right]\left(\frac{b}{d}-\hat{N}(\alpha)\right) \\
& \hat{P}(\alpha)=\left[\frac{d}{\alpha} \frac{\theta(\alpha)}{\sigma(\alpha)}\right]\left(\frac{b}{d}-\hat{N}(\alpha)\right) \\
& \hat{I}(\alpha)=\hat{P}(\alpha) \sigma(\alpha) / \theta(\alpha)
\end{aligned}
$$

These are the same as equations (12) except that we have not substituted (10) so that $g$ is arbitrary. The first three equations are closed so that the last of these equations will not be needed. Because $f$ and $g$ are unknown, these equations cannot be solved explicitly. Substituting (23b) and (23c) into (23a) gives a single equation for $\hat{N}(\alpha)$, and once it is solved, $\hat{S}(\alpha)$ and $\hat{P}(\alpha)$ follow immediately.

We shall also need the derivatives $\hat{N}^{\prime}(\alpha), \hat{S}^{\prime}(\alpha)$ and $\hat{P}^{\prime}(\alpha)$. The latter two are immediate from $(23 \mathrm{~b})$ and $(23 \mathrm{c})$ and hold for all $\alpha$ :

$$
\begin{aligned}
& \hat{S}^{\prime}(\alpha)=\hat{N}^{\prime}(\alpha)\left[\frac{\alpha+d+\rho(\alpha)}{\alpha}\right]-\left(\frac{b}{d}-\hat{N}(\alpha)\right)\left[\frac{d+\rho(\alpha)}{\alpha}\right]^{\prime} \\
& \hat{P}^{\prime}(\alpha)=\left[\frac{d}{\alpha} \frac{\theta(\alpha)}{\sigma(\alpha)}\right]^{\prime}\left(\frac{b}{d}-\hat{N}(\alpha)\right)-\hat{N}^{\prime}(\alpha)\left[\frac{d}{\alpha} \frac{\theta(\alpha)}{\sigma(\alpha)}\right]
\end{aligned}
$$


To obtain $\hat{N}^{\prime}$ at the singularity, differentiate (23a) and use (6) and (23a) to arrive at

$$
\frac{\hat{S}^{\prime}\left(\alpha^{*}\right)}{S^{*}}+S^{*}\left[D\left(\alpha^{*}\right)\left(\frac{f(N)}{N}\right)_{N=N^{*}}^{\prime} \hat{N}^{\prime}\left(\alpha^{*}\right)+E\left(\alpha^{*}\right)\left(\frac{g(P)}{P}\right)_{P=P^{*}}^{\prime} \hat{P}^{\prime}\left(\alpha^{*}\right)\right]=0
$$

where starred densities are the equilibrium densities of the singular strain $\alpha^{*}$ (i.e., $S^{*}=$ $\left.\hat{S}\left(\alpha^{*}\right), N^{*}=\hat{N}\left(\alpha^{*}\right), P^{*}=\hat{P}\left(\alpha^{*}\right)\right)$. Substituting (24a) and (24b) yields a linear equation for $\hat{N}^{\prime}\left(\alpha^{*}\right)$.

\subsection{Non-degeneracy condition for constructing $g_{0}$}

$g_{0}$ is defined in (13) as the parametric curve $\left(\hat{P}(\alpha), g_{0}(\hat{P}(\alpha))=\left(\hat{P}(\alpha), c \hat{P}(\alpha) \frac{f(\hat{N}(\alpha))}{\hat{N}(\alpha)}\right)\right.$. This parametric curve need not be a function (cf. Figure 1), but it is a function over any interval of $\alpha$ in which $\hat{P}^{\prime}(\alpha)$ does not vanish. By continuity, there exists such an interval $\left(\alpha_{\min }, \alpha_{\max }\right)$ around the singularity $\alpha^{*}$ if $\hat{P}^{\prime}\left(\alpha^{*}\right) \neq 0$.

In this section, we calculate the derivatives $\hat{N}^{\prime}\left(\alpha^{*}\right), \hat{S}^{\prime}\left(\alpha^{*}\right)$ and $\hat{P}^{\prime}\left(\alpha^{*}\right)$ assuming $g(P)=g_{0}(P)$; these derivatives will be used also in the remainder of this appendix. The condition for $\hat{P}^{\prime}\left(\alpha^{*}\right) \neq 0$ appears in (32).

By differentiating (13) with respect to $\alpha$, we have

$$
\left(\frac{g_{0}(P)}{P}\right)^{\prime} \hat{P}^{\prime}(\alpha)=c\left(\frac{f(N)}{N}\right)^{\prime} \hat{N}^{\prime}(\alpha)
$$

Substituting this into (25) and using (23a) and (13) leads to

$$
\frac{\hat{S}^{\prime}\left(\alpha^{*}\right)}{S^{*}} \frac{f\left(N^{*}\right)}{N^{*}}+\left(\frac{f(N)}{N}\right)_{N=N^{*}}^{\prime} \hat{N}^{\prime}\left(\alpha^{*}\right)=0
$$

and with (24a) and (23b) this yields

$$
\frac{\hat{N}^{\prime}\left(\alpha^{*}\right)}{N^{*}}=\frac{\frac{f\left(N^{*}\right)}{N^{*}}\left(\frac{b}{d}-N^{*}\right)\left[\frac{d+\rho(\alpha)}{\alpha}\right]_{\alpha^{*}}^{\prime}}{\frac{f\left(N^{*}\right)}{N^{*}} \frac{b}{d}\left[\frac{d+\rho\left(\alpha^{*}\right)}{\alpha^{*}}\right]+f^{\prime}\left(N^{*}\right) S^{*}}
$$

Notice that since $N^{*}<\frac{b}{d}$ for all positive virulence and by assumption $\rho^{\prime}(\alpha) \leq 0$, the numerator of (28) is negative; and because $f$ is assumed to be nondecreasing, the denominator is strictly positive. $\hat{N}^{\prime}\left(\alpha^{*}\right)$ is therefore always negative.

By substituting (28) into (24a) and using (23b) and (28), we obtain

$$
\frac{\hat{S}^{\prime}\left(\alpha^{*}\right)}{S^{*}}=\frac{\hat{N}^{\prime}\left(\alpha^{*}\right)}{N^{*}}\left[1-\frac{f^{\prime}\left(N^{*}\right)}{\frac{f\left(N^{*}\right)}{N^{*}}}\right]
$$


Because $\hat{N}^{\prime}\left(\alpha^{*}\right)$ is negative, $\hat{S}^{\prime}\left(\alpha^{*}\right)$ is negative if and only if

$$
f^{\prime}\left(N^{*}\right)<\frac{f\left(N^{*}\right)}{N^{*}}
$$

Note that this condition holds if $f$ is concave with $f(0) \geq 0$.

From (24b) and (23c), we have

$$
\frac{\hat{P}^{\prime}\left(\alpha^{*}\right)}{P^{*}}=\frac{\left[\frac{d}{\alpha} \frac{\theta(\alpha)}{\sigma(\alpha)}\right]_{\alpha^{*}}^{\prime}}{\left[\frac{d}{\alpha} \frac{\theta(\alpha)}{\sigma(\alpha)}\right]_{\alpha^{*}}}-\frac{\hat{N}^{\prime}\left(\alpha^{*}\right)}{\left(\frac{b}{d}-N^{*}\right)}
$$

Substituting (28) and rearranging shows that $\hat{P}^{\prime}\left(\alpha^{*}\right) \neq 0$ if and only if

$$
\left[\frac{d}{\alpha} \frac{\theta(\alpha)}{\sigma(\alpha)}\right]_{\alpha^{*}}^{\prime} \neq\left[\frac{d}{\alpha} \frac{\theta(\alpha)}{\sigma(\alpha)}\right]_{\alpha^{*}} \frac{f\left(N^{*}\right)\left[\frac{d+\rho(\alpha)}{\alpha}\right]_{\alpha^{*}}^{\prime}}{\left.N^{*}\right) \frac{b}{d}\left[\frac{d+\rho\left(\alpha^{*}\right)}{\alpha^{*}}\right]+f^{\prime}\left(N^{*}\right) S^{*}}
$$

Note that since $\hat{N}^{\prime}\left(\alpha^{*}\right)$ is negative in $(24 \mathrm{~b})$,

$$
\left[\frac{d}{\alpha} \frac{\theta(\alpha)}{\sigma(\alpha)}\right]_{\alpha^{*}}^{\prime}=\frac{d\left[\theta^{\prime}\left(\alpha^{*}\right) \alpha^{*} \sigma\left(\alpha^{*}\right)-\theta\left(\alpha^{*}\right) \sigma\left(\alpha^{*}\right)-\theta\left(\alpha^{*}\right) \alpha^{*} \sigma^{\prime}\left(\alpha^{*}\right)\right]}{\left(\alpha^{*} \sigma\left(\alpha^{*}\right)\right)^{2}} \geq 0
$$

ensures that $\hat{P}^{\prime}\left(\alpha^{*}\right)$ is positive. This sufficient condition holds for example if $\theta(\alpha) / \sigma(\alpha)$ is a convex function of $\alpha$ with $\theta(0)=0$. If one assumes realistically that the shedding rate is an increasing function of virulence $\left(\theta^{\prime}(\alpha)>0\right)$ and the decay rate is nonincreasing $\left(\sigma^{\prime}(\alpha) \leq 0\right)$, then (33) holds if $\theta$ is linear or convex with $\theta(0)=0$ (such that $\left.\theta^{\prime}(\alpha) \alpha \geq \theta(\alpha)\right)$.

\subsection{Conditions guaranteeing that $g_{0}$ is increasing}

Biological considerations dictate that the incidence function $g$ should be increasing (or at least non-decreasing). In this section, we investigate the conditions under which $g_{0}$ is an increasing function in the vicinity of $P^{*}$.

Assuming $\hat{P}^{\prime}\left(\alpha^{*}\right) \neq 0$, from (26) we obtain

$$
g_{0}^{\prime}\left(P^{*}\right)=c\left[\frac{f\left(N^{*}\right)}{N^{*}}+P^{*} \frac{\hat{N}^{\prime}\left(\alpha^{*}\right)}{\hat{P}^{\prime}\left(\alpha^{*}\right)}\left(\frac{f(N)}{N}\right)_{N=N^{*}}^{\prime}\right]
$$

which, by substituting $\left(\frac{f(N)}{N}\right)_{N=N^{*}}^{\prime}$ from (27) and using (13), simplifies to

$$
g_{0}^{\prime}\left(P^{*}\right)=\frac{g_{0}\left(P^{*}\right)}{P^{*}}\left[1-\frac{P^{*}}{S^{*}} \frac{\hat{S}^{\prime}\left(\alpha^{*}\right)}{\hat{P}^{\prime}\left(\alpha^{*}\right)}\right]
$$


$g_{0}^{\prime}\left(P^{*}\right)$ is obviously positive if $\hat{S}^{\prime}\left(\alpha^{*}\right) / S^{*}$ and $\hat{P}^{\prime}\left(\alpha^{*}\right) / P^{*}$, given respectively in $(29)$ and (31), have different signs; (30) together with (33) are therefore a simple set of sufficient conditions. These are however somewhat restrictive: on the one hand, (30) limits the choice of $f$ locally; and on the other hand, (33) is not necessarily satisfied by biologically plausible trade-offs.

To give necessary and sufficient conditions for $g_{0}^{\prime}\left(P^{*}\right)>0$, note that $g_{0}^{\prime}\left(P^{*}\right)$ changes

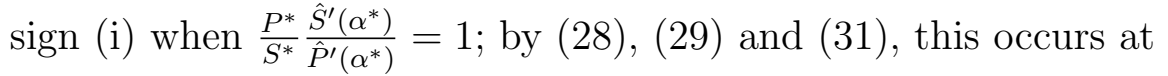

$$
\left[\frac{d}{\alpha} \frac{\theta(\alpha)}{\sigma(\alpha)}\right]_{\alpha^{*}}^{\prime}=\left[\frac{d}{\alpha} \frac{\theta(\alpha)}{\sigma(\alpha)}\right]_{\alpha^{*}} \frac{\left[f\left(N^{*}\right)+\left(\frac{b}{d}-N^{*}\right)\left(\frac{f\left(N^{*}\right)}{N^{*}}-f^{\prime}\left(N^{*}\right)\right)\right]\left[\frac{d+\rho(\alpha)}{\alpha}\right]_{\alpha^{*}}^{\prime}}{\frac{f\left(N^{*}\right)}{N^{*}} \frac{b}{d}\left[\frac{d+\rho\left(\alpha^{*}\right)}{\alpha^{*}}\right]+f^{\prime}\left(N^{*}\right) S^{*}}
$$

and (ii) when $\hat{P}^{\prime}\left(\alpha^{*}\right)=0$ and changes sign, which occurs when (32) is violated. For either sufficiently large positive or large negative values of $\left[\frac{d}{\alpha} \frac{\theta(\alpha)}{\sigma(\alpha)}\right]_{\alpha^{*}}^{\prime}, \hat{P}^{\prime}\left(\alpha^{*}\right)$ is large in absolute value (cf. (24b)), and therefore $g_{0}^{\prime}\left(P^{*}\right)$ is positive irrespectively of the signs of $\hat{S}^{\prime}\left(\alpha^{*}\right)$ and $\hat{P}^{\prime}\left(\alpha^{*}\right)$. Thus $g_{0}^{\prime}\left(P^{*}\right)$ is negative inbetween the thresholds (i) and (ii), and positive outside. Threshold (i) in (36) is lower than (ii) in (32) if and only if $f^{\prime}\left(N^{*}\right)<\frac{f\left(N^{*}\right)}{N^{*}}$ (recall that $\frac{b}{d}-N^{*}>0$ and $\left.\left[\frac{d+\rho(\alpha)}{\alpha}\right]_{\alpha^{*}}^{\prime}<0\right)$.

In summary, $g_{0}^{\prime}\left(P^{*}\right)$ is positive if and only if

$$
\frac{\left[\frac{d}{\alpha} \frac{\theta(\alpha)}{\sigma(\alpha)}\right]_{\alpha^{*}}^{\prime}}{\left[\frac{d}{\alpha} \frac{\theta(\alpha)}{\sigma(\alpha)}\right]_{\alpha^{*}}} \notin[m, M]
$$

where $m$ and $M$ are given as follows:

(a) if $f^{\prime}\left(N^{*}\right)<\frac{f\left(N^{*}\right)}{N^{*}}$ then

$$
\begin{aligned}
m & =\frac{\left[f\left(N^{*}\right)+\left(\frac{b}{d}-N^{*}\right)\left(\frac{f\left(N^{*}\right)}{N^{*}}-f^{\prime}\left(N^{*}\right)\right)\right]\left[\frac{d+\rho(\alpha)}{\alpha}\right]_{\alpha^{*}}^{\prime}}{\frac{f\left(N^{*}\right)}{N^{*}} \frac{b}{d}\left[\frac{d+\rho\left(\alpha^{*}\right)}{\alpha^{*}}\right]+f^{\prime}\left(N^{*}\right) S^{*}} \\
M & =\frac{f\left(N^{*}\right)\left[\frac{d+\rho(\alpha)}{\alpha}\right]_{\alpha^{*}}^{\prime}}{\frac{f\left(N^{*}\right)}{N^{*}} \frac{b}{d}\left[\frac{d+\rho\left(\alpha^{*}\right)}{\alpha^{*}}\right]+f^{\prime}\left(N^{*}\right) S^{*}}
\end{aligned}
$$

(b) if $f^{\prime}\left(N^{*}\right)>\frac{f\left(N^{*}\right)}{N^{*}}$ then $m$ and $M$ are reversed.

If $f^{\prime}\left(N^{*}\right)=\frac{f\left(N^{*}\right)}{N^{*}}$ then $m$ and $M$ coincide and $g_{0}^{\prime}\left(P^{*}\right)$ is always positive when it exists, i.e., when (32) is satisfied. 


\subsection{Transversality condition for unfolding $\mathcal{M}=0$}

In this section, we investigate how $\mathcal{M}$ changes with changing $g^{\prime}\left(P^{*}\right)$. Let $s$ denote the slope of the incidence function $g$ at the singularity (i.e., $s=g^{\prime}\left(P^{*}\right)$ ) and let $s_{0}$ be the slope of $g_{0}$ at the same point $\left(s_{0}=g_{0}^{\prime}\left(P^{*}\right)\right)$. In (8), $\mathcal{M}$ is given as

$$
\mathcal{M}=S^{*}\left[D^{\prime}\left(\alpha^{*}\right)\left(\frac{f(N)}{N}\right)_{N=N^{*}}^{\prime} \hat{N}^{\prime}\left(\alpha^{*}\right)+E^{\prime}\left(\alpha^{*}\right)\left(\frac{g(P)}{P}\right)_{P=P^{*}}^{\prime} \hat{P}^{\prime}\left(\alpha^{*}\right)\right]
$$

Changing the derivative $s=g^{\prime}\left(P^{*}\right)$ [while leaving the value of $g\left(P^{*}\right)$ unchanged] does not affect the equilibrium densities of strain $\alpha^{*}$ so that $N^{*}, S^{*}$ and $P^{*}$ remain the same; and it does not affect the singularity condition (6) so that $\alpha^{*}$ remains singular. The derivatives $\hat{N}^{\prime}\left(\alpha^{*}\right)$ and $\hat{P}^{\prime}\left(\alpha^{*}\right)$ however do change. Using

$$
\left(\frac{g(P)}{P}\right)_{P=P^{*}}^{\prime}=\frac{1}{P^{*}}\left[s-\frac{g\left(P^{*}\right)}{P^{*}}\right]
$$

and differentiating (40) with respect to $s$ yields

$$
\begin{aligned}
\frac{d \mathcal{M}}{d s}= & S^{*}\left[D^{\prime}\left(\alpha^{*}\right)\left(\frac{f(N)}{N}\right)_{N=N^{*}}^{\prime} \frac{d \hat{N}^{\prime}\left(\alpha^{*}\right)}{d s}+E^{\prime}\left(\alpha^{*}\right)\left(\frac{g(P)}{P}\right)_{P=P^{*}}^{\prime} \frac{d \hat{P}^{\prime}\left(\alpha^{*}\right)}{d s}+\right. \\
& \left.+E^{\prime}\left(\alpha^{*}\right) \frac{\hat{P}^{\prime}\left(\alpha^{*}\right)}{P^{*}}\right]
\end{aligned}
$$

To obtain the derivatives $\frac{d \hat{N}^{\prime}\left(\alpha^{*}\right)}{d s}$ and $\frac{d \hat{P}^{\prime}\left(\alpha^{*}\right)}{d s}$, differentiate (25) implicitly with respect to $s$ using (41). This yields

$$
\begin{aligned}
& \frac{d \hat{S}^{\prime}\left(\alpha^{*}\right)}{d s}+S^{* 2}\left[D\left(\alpha^{*}\right)\left(\frac{f(N)}{N}\right)_{N=N^{*}}^{\prime} \frac{d \hat{N}^{\prime}\left(\alpha^{*}\right)}{d s}+\right. \\
& \left.+E\left(\alpha^{*}\right)\left(\frac{g(P)}{P}\right)_{P=P^{*}}^{\prime} \frac{d \hat{P}^{\prime}\left(\alpha^{*}\right)}{d s}+E\left(\alpha^{*}\right) \frac{\hat{P}^{\prime}\left(\alpha^{*}\right)}{P^{*}}\right]=0
\end{aligned}
$$

Substituting

$$
\begin{aligned}
\frac{d \hat{S}^{\prime}\left(\alpha^{*}\right)}{d s} & =\left[\frac{\alpha^{*}+d+\rho\left(\alpha^{*}\right)}{\alpha^{*}}\right] \frac{d \hat{N}^{\prime}\left(\alpha^{*}\right)}{d s} \\
\frac{d \hat{P}^{\prime}\left(\alpha^{*}\right)}{d s} & =-\left[\frac{d}{\alpha^{*}} \frac{\theta\left(\alpha^{*}\right)}{\sigma\left(\alpha^{*}\right)}\right] \frac{d \hat{N}^{\prime}\left(\alpha^{*}\right)}{d s}
\end{aligned}
$$

obtained directly from $(24 a, b)$, we arrive at

$$
\begin{aligned}
& \frac{d \hat{N}^{\prime}\left(\alpha^{*}\right)}{d s}\left[\frac{\alpha^{*}+d+\rho\left(\alpha^{*}\right)}{\alpha^{*}}+S^{* 2}\left(D\left(\alpha^{*}\right)\left(\frac{f(N)}{N}\right)_{N=N^{*}}^{\prime}-\right.\right. \\
& \left.\left.-E\left(\alpha^{*}\right)\left(\frac{g(P)}{P}\right)_{P=P^{*}}^{\prime} \frac{d}{\alpha^{*}} \frac{\theta\left(\alpha^{*}\right)}{\sigma\left(\alpha^{*}\right)}\right)\right]=-E\left(\alpha^{*}\right) S^{* 2} \frac{\hat{P}^{\prime}\left(\alpha^{*}\right)}{P^{*}}
\end{aligned}
$$


which, using (25) and (24a,b) again, simplifies to

$$
\begin{aligned}
& \frac{d \hat{N}^{\prime}\left(\alpha^{*}\right)}{d s}\left[\left(\frac{b}{d}-N^{*}\right)\left[\frac{d}{\alpha} \frac{\theta(\alpha)}{\sigma(\alpha)}\right]_{\alpha^{*}}^{\prime}\left(1-S^{* 2} E\left(\alpha^{*}\right)\left(\frac{g(P)}{P}\right)_{P=P^{*}}^{\prime}\right)\right]= \\
& =-E\left(\alpha^{*}\right) S^{* 2} \frac{\hat{P}^{\prime}\left(\alpha^{*}\right)}{P^{*}} \hat{N}^{\prime}\left(\alpha^{*}\right)
\end{aligned}
$$

This is a simple linear equation for $\frac{d \hat{N}^{\prime}\left(\alpha^{*}\right)}{d s}$; and $\frac{d \hat{P}^{\prime}\left(\alpha^{*}\right)}{d s}$ follows from (44b) immediately.

With $g(P)=g_{0}(P), \alpha^{*}$ is optimal so that neither mutual invasibility nor mutual exclusion occurs near the singularity; and therefore at $s=s_{0}, \mathcal{M}$ is zero. By (40) and (24b) this implies

$$
\begin{aligned}
& D^{\prime}\left(\alpha^{*}\right)\left(\frac{f(N)}{N}\right)_{N=N^{*}}^{\prime}-E^{\prime}\left(\alpha^{*}\right)\left(\frac{g_{0}(P)}{P}\right)_{P=P^{*}}^{\prime}\left[\frac{d}{\alpha^{*}} \frac{\theta\left(\alpha^{*}\right)}{\sigma\left(\alpha^{*}\right)}\right]= \\
& =-\frac{E^{\prime}\left(\alpha^{*}\right)}{\hat{N}^{\prime}\left(\alpha^{*}\right)}\left(\frac{g_{0}(P)}{P}\right)_{P=P^{*}}^{\prime}\left(\frac{b}{d}-N^{*}\right)\left[\frac{d}{\alpha} \frac{\theta(\alpha)}{\sigma(\alpha)}\right]_{\alpha^{*}}^{\prime}
\end{aligned}
$$

This we use to simplify $d \mathcal{M} / d s$ at $s=s_{0}$. From (42) and (44b) we have

$$
\begin{aligned}
\frac{d \mathcal{M}}{d s}= & S^{*}\left[E^{\prime}\left(\alpha^{*}\right) \frac{\hat{P}^{\prime}\left(\alpha^{*}\right)}{P^{*}}+\frac{d \hat{N}^{\prime}\left(\alpha^{*}\right)}{d s} \times\right. \\
& \left.\times\left(D^{\prime}\left(\alpha^{*}\right)\left(\frac{f(N)}{N}\right)_{N=N^{*}}^{\prime}-E^{\prime}\left(\alpha^{*}\right)\left(\frac{g(P)}{P}\right)_{P=P^{*}}^{\prime}\left[\frac{d}{\alpha^{*}} \frac{\theta\left(\alpha^{*}\right)}{\sigma\left(\alpha^{*}\right)}\right]\right)\right]
\end{aligned}
$$

and with (47) this becomes

$$
\begin{aligned}
\left.\frac{d \mathcal{M}}{d s}\right|_{s=s_{0}}= & S^{*}\left[E^{\prime}\left(\alpha^{*}\right) \frac{\hat{P}^{\prime}\left(\alpha^{*}\right)}{P^{*}}-\left.\frac{d \hat{N}^{\prime}\left(\alpha^{*}\right)}{d s}\right|_{s=s_{0}} \times\right. \\
& \left.\times \frac{E^{\prime}\left(\alpha^{*}\right)}{\hat{N}^{\prime}\left(\alpha^{*}\right)}\left(\frac{g_{0}(P)}{P}\right)_{P=P^{*}}^{\prime}\left(\frac{b}{d}-N^{*}\right)\left[\frac{d}{\alpha} \frac{\theta(\alpha)}{\sigma(\alpha)}\right]_{\alpha^{*}}^{\prime}\right]= \\
= & \frac{S^{*} E^{\prime}\left(\alpha^{*}\right) \frac{\hat{P}^{\prime}\left(\alpha^{*}\right)}{P^{*}}}{1+E\left(\alpha^{*}\right) S^{*} \frac{g_{0}\left(P^{*}\right)}{P^{*}} \frac{\hat{S}^{\prime}\left(\alpha^{*}\right)}{\hat{P}^{\prime}\left(\alpha^{*}\right)}}
\end{aligned}
$$

where in the last step we substituted $\frac{d \hat{N}^{\prime}\left(\alpha^{*}\right)}{d s}$ from equation (46), using (41) with $s_{0}=$ $\frac{g_{0}\left(P^{*}\right)}{P^{*}}\left[1-\frac{P^{*}}{S^{*}} \hat{S}^{\prime}\left(\alpha^{*}\right)\right]$ (cf. (35). In (49), the derivatives $\hat{N}^{\prime}\left(\alpha^{*}\right), \hat{S}^{\prime}\left(\alpha^{*}\right)$ and $\hat{P}^{\prime}\left(\alpha^{*}\right)$ are from equations (28), (29) and (31), respectively.

Recall that $E^{\prime}\left(\alpha^{*}\right)$ cannot be zero and that the non-degeneracy condition (32) guarantees $\hat{P}^{\prime}\left(\alpha^{*}\right) \neq 0$. $\left.\frac{d \mathcal{M}}{d s}\right|_{s=s_{0}}$ therefore does not vanish, and perturbing $s=g^{\prime}\left(P^{*}\right)$ from $s_{0}=g_{0}^{\prime}\left(P^{*}\right)$ will unfold the degeneracy $\mathcal{M}=0$. To obtain $\mathcal{M}<0$ so that there is mutual invasibility near the singularity, the sign of $s-s_{0}$ should be opposite to that of (49). 